\title{
Longitudinal Feedback in LEP
}

\author{
J. P. Boiteux, P. Brown, E. Ciapala, H. Frischholz, G. Geschonke, J. C. Juillard and E. Peschardt \\ CERN \\ CH-1211 Geneva 23
}

\begin{abstract}
Dipole coupled bunch oscillations were observed at an early stage of LEP commissioning for currents above about $150 \mu \mathrm{A}$ per bunch. An improvised feedback system, acting on the phase of some of the accelerating cavities was developed and has been in operation for about three years. However, due to the small bandwidth of the RF cavities this system can only be used with four bunches or less per beam. With plans for eight bunch operation (the Pretzel scheme) the construction of a dedicated longitudinal feedback system was approved in 1991. The system operates at $999.95 \mathrm{MHz}$ with phase modulation of a $200 \mathrm{~kW}$ klystron feeding four sevencell cavities. The necessary bandwidth of $260 \mathrm{kHz}$ is obtained by heavy over-coupling. With a total cavity voltage of $1.9 \mathrm{MV}$ a damping rate of about $450 \mathrm{~s}^{-1}$ is obtained with phase excursions of one radian. The system has been in routine operation since July 1992 with a feedback cavity voltage of $1.2 \mathrm{MV}$ and a damping rate of about $100 \mathrm{~s}^{-1}$. Longitudinal feedback eases operation and usually increases the maximum currents which can be accumulated.
\end{abstract}

\section{INTRODUCTION}

Longitudinal instabilities were not expected in LEP before the current was approaching the design value of $0.75 \mathrm{~mA}$ per bunch. However, during the commissioning of the collider in 1989, coupled dipole oscillations were seen at currents above about $0.15 \mathrm{~mA}$ per bunch when LEP was operated with four bunches per beam. They were cured with an improvised feedback system [1] which acts on the beams via some of the accelerating cavities. A fundamental limitation of this system is the narrow bandwidth of these cavities. It can therefore only be used for beams with four bunches or less. With plans for eight bunch operation in LEP [2], a longitudinal feedback system using dedicated cavities was therefore prepared and finally approved in March 1991.

\section{SYSTEM REQUIREMENTS}

The cost of a high power RF system is mainly determined by the RF power requirement which is calculated from the necessary bandwidth and voltage. High frequency operation is advantageous because for a given bandwidth, cavity length and voltage, the required power decreases with frequency.

\section{A. Bandwidth}

In order for the energy error of each bunch to be corrected individually, the cavity field has to change in the time interval between the passage of two bunches. Therefore the longer this time interval, the higher the cavity Q-value which can be used. In the case of two counter-rotating beams of eight bunches this time interval would be maximum if the feedback cavities were located $1 / 16$ th of the circumference from a collision point. In LEP this is not possible and the only free space available is located $435 \mathrm{~m}$ from the intersection points. At this position the minimum time between bunch passages is $2.9 \mu \mathrm{s}$. Assuming that in this time interval the cavity field should reach $90 \%$ of the set value, the required cavity filling time is $1.26 \mu$ s with a resulting bandwidth of about $260 \mathrm{kHz}$.

With this bandwidth the system can be used with any even number of bunches up to 12 and if the cavity bandwidth is increased to $300 \mathrm{kHz}, 18$ bunch operation is also possible.

\section{B. Frequency}

By choosing a frequency of about $1 \mathrm{GHz}$, four cavities of a type developed and constructed by DESY can be used. Within their tuning range the exact frequency is determined by the revolution frequency and the number of bunches in LEP. If the harmonic number is a multiple of 360 the feedback system allows operation for all possible number of bunches which have been studied. The harmonic number is then found to be 88920 and the frequency $999.9497 \mathrm{MHz}\left(247 / 87 \mathrm{f}_{\mathrm{RF}}\right)$. Using a frequency which is not an harmonic of the LEP RF frequency of $352.209 \mathrm{MHz}$ complicates the frequency generation system but the functioning of the system is not effected.

\section{Cavity Voltage}

Four seven-cell cavities are used for the system. The $260 \mathrm{kHz}$ bandwidth is obtained by lowering the loaded Q-value with strong over-coupling. A cavity voltage of $625 \mathrm{kV}$ is expected for $40 \mathrm{~kW}$ at the input coupler. With a generator power of $200 \mathrm{~kW}$ and $20 \%$ wave guide losses the total voltage for four cavities is about $2.5 \mathrm{MV}$.

For phase modulation with a modulation index or $\pi / 2$ the relationship between feedback voltage, $U_{F B}$ and feedback damping rate, $\alpha_{F B}$ is [3]:

$$
U_{F B}=\frac{2 \alpha_{F B} E_{0}}{e f_{\text {reo }}}\left(\frac{\Delta E}{E_{0}}\right)
$$

where $f_{r e v}$ is the LEP revolution frequency, $E_{0}$ the energy and $\triangle E / E_{0}$ the maximum likely coherent energy oscillation. For $E_{0}=20 \mathrm{GeV}$ and $\Delta E / E_{0}=10^{-3}$ and if for linearity reasons the phase excursions are limited to one radian the feedback voltage is:

$$
U_{F B}=4.2 \times 10^{3} \alpha_{F B}
$$

giving a maximum damping rate of $600 \mathrm{~s}^{-1}$. 


\section{SYSTEM DESCRIPTION}

The dipole oscillations are detected by the system which was developed for the first feedback system [1] where the signal from a sum pick-up is phase compared with the RF frequency. The only addition required is a time multiplexing scheme which selects the signals from the positron and electron bunch oscillation detector in such a way that the signal from a particular bunch is applied to the cavities during the passage of the same bunch one turn later.

A schematic block diagram is shown in Figure 1. The feedback signal is applied to a fast phase shifter. Phase modulation has been chosen in order to operate the klystron with constant RF power. The drive level dependent phase shift in the klystron is then eliminated and the cavity tuning system is not disturbed.

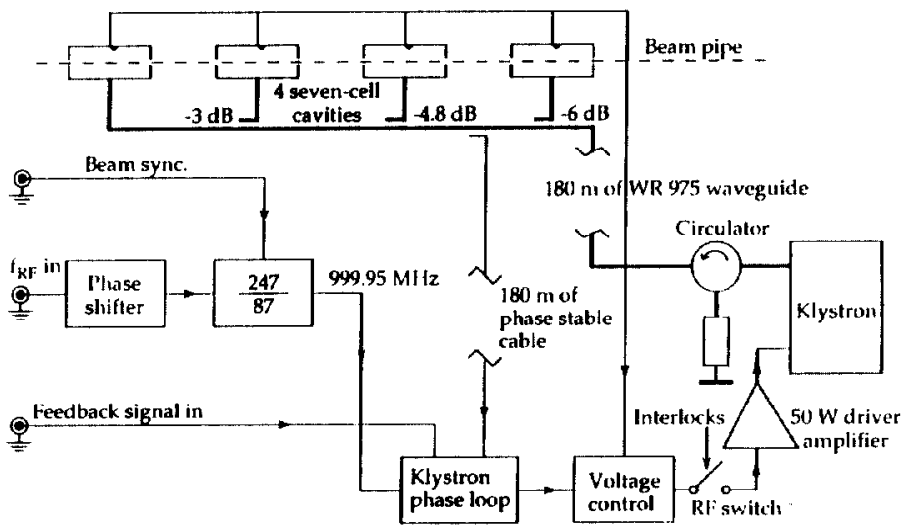

Figure 1. Block diagram of the feedback system.

The $1 \mathrm{GHz}$ signal is generated in a voltage controlled crystal oscillator locked to the RF frequency (Figure 2). In addition because the frequency of the system is not a harmonic of the RF frequency, synchronisation to one of the beams is required. The latter is obtained from a sum pick-up signal processed to detect the beam polarity and with flip-flops (FF) used to reset a counter. When available, the positron bunches are used for this synchronisation, otherwise the system switches automatically to the electron bunches.

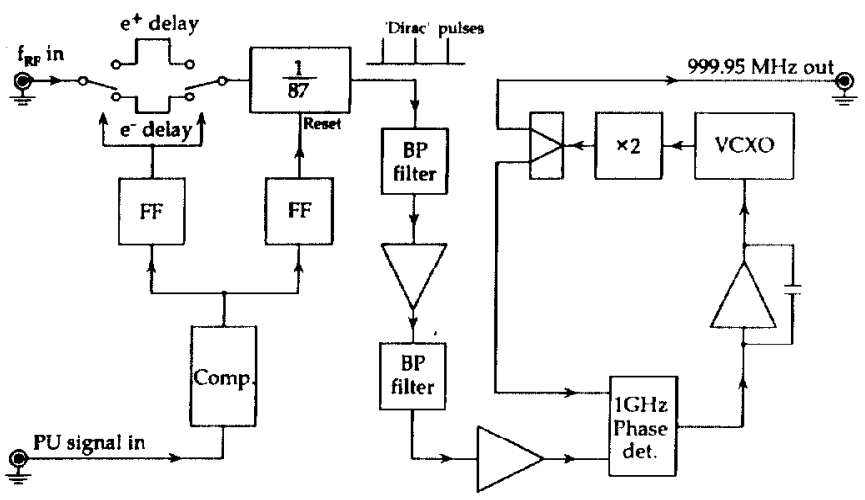

Figure 2. Schematic diagram of the frequency generator.
The distance between the power generation system and the cavities is about $180 \mathrm{~m}$. The signal for the klystron phase loop which compensates for phase variations in the power generation system is returned from the cavity end through a special phase stable cable which has a delay stability better than $2 \mathrm{ppm} /{ }^{\circ} \mathrm{C}$ at $500 \mathrm{MHz}$.

\section{B. RF Power Generation and Distribution}

The cavities are powered by a $200 \mathrm{~kW}$ klystron, operating at $999.95 \mathrm{MHz}$. The $-1 \mathrm{~dB}$ output bandwidth of this klystron is $\pm 2 \mathrm{MHz}$. A $60 \mathrm{~W}$ solid state amplifier drives the klystron via a short length of coaxial cable. The amplifier output is protected from any load mismatch by a circulator.

From the over-coupled cavities about $50 \%$ of the incident power is reflected. In order to protect the klystron against these reflections a waveguide differential phase shift circulator is inserted in its output line. The rated power handling capability of the circulator is $400 \mathrm{~kW}$ with up to $75 \%$ reflections at any RF phase angle. Ports three and four are terminated by coaxial water loads with maximum RF power dissipation ratings of 100 and $40 \mathrm{~kW}$ respectively.

The RF power is transmitted to the cavities via WR975 waveguides and three power splitters which comprise shortslot waveguide hybrids with coupling factors of $-6,-4.77$ and $-3 \mathrm{~dB}$, thus ensuring equal input power to all the cavities. The insertion loss of the $180 \mathrm{~m}$ long waveguide line is about $1 \mathrm{~dB}$, which means that at $200 \mathrm{~kW}$ incident and $100 \mathrm{~kW}$ reflected RF power about $56 \mathrm{~kW}$ is dissipated in the waveguide walls. In order not to overload the air ventilation system of the LEP tunnel the waveguides must be water cooled. An extruded $\mathrm{Al}$ profile with a cooling water channel was therefore bolted on the centre line of both wide sides of each waveguide. At a water flow of $10 \mathrm{l} / \mathrm{min}$ in both cooling channels about $80 \%$ of the power dissipated in the waveguides is absorbed by the water, resulting in an average waveguide temperature increase of only $6^{\circ} \mathrm{C}$ at the above mentioned $\mathrm{RF}$ power flows.

In April 1993 the vertical $200 \mathrm{~kW}$ Philips klystron V109SK was replaced by the horizontal EEV model $\mathrm{K} 3440 \mathrm{C}$ which provides about twice the output power of the previously installed one.

\section{Cavities}

DESY generously supplied CERN with four copper accelerating cavities. Each cavity consists of seven pill box cells without nose cones, cell coupling via the beam hole. They were fully equipped with tuners in the third and fifth cells, centre-cell power coupler and field probes.

The required bandwidth was achieved by strongly overcoupling the power coupler. This was done by increasing its penetration by $25 \mathrm{~mm}$ and by altering the field distribution by means of a fixed tuner of $50 \mathrm{~mm}$ diameter, penetrating $21 \mathrm{~mm}$ into the centre cell. The loaded Q-values thus obtained are between 3250 and 3950, giving a bandwidth between 253 and $304 \mathrm{kHz}$. The unloaded Q-value was measured to be 27000 . 
After the modifications the accelerating field flatness in the seven cells was within $\pm 8 \%$.

The RF input power couplers developed by DESY are a coaxial type with a brazed ceramic disk. The power handling capability was increased from its original design value of $40 \mathrm{~kW}$ to $70 \mathrm{~kW}$ by modifications to the air cooling system and by modifying the RF contacts on the inner conductor.

For the tuning system, wherever possible, equipment built for the LEP accelerating cavities was used. The system uses the phase between the forward travelling wave taken from a directional coupler in the wave guide feeder line directly in front of the cavities and the centre cell field probe for tuning and the amplitude ratio between the third and the fifth cell probes for field balancing. The tuners are driven with fivephase stepping motors. The tuning range is about $4.1 \mathrm{MHz}$.

Before installation in LEP all the cavities were individually conditioned to an RF power of $70 \mathrm{~kW}$.

\section{SYSTEM PERFORMANCE}

The phase of the feedback cavity voltage was adjusted with respect to the $352 \mathrm{MHz} R \mathrm{R}$ voltage by maximising the synchrotron frequency. From the increase of this frequency when the feedback voltage is switched on the actual voltage is found. For some reasons, possibly bad power calibrations, this voltage is about $20 \%$ lower than expected.

$$
A=2 V \quad 6.25 \mathrm{~ms}
$$

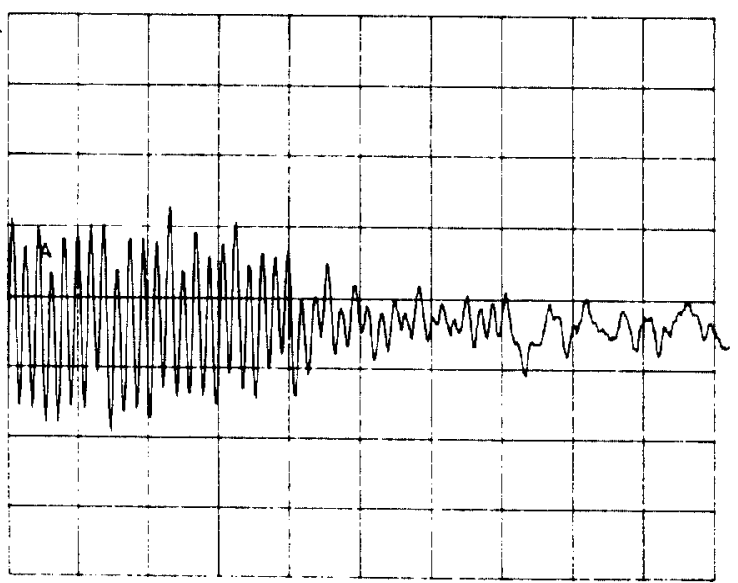

Figure 3. The decrease in amplitude of the dipole bunch oscillations when the feedback is switched on. The damping time constant was about $10 \mathrm{~ms}$ with a cavity voltage of 1.6 MV and a current of $100 \mu \mathrm{A}$ per bunch.

The modulation was then added and after a few adjustments the system damped all eight modes of the two eight bunch counter-rotating beams. Figure 3 shows the decrease in oscillation amplitude for a particular bunch when feedback is switched on. In this case the damping time was about $10 \mathrm{~ms}$ with a feedback cavity voltage of $1.6 \mathrm{MV}$. The rise time of the oscillations was for the same bunch about $50 \mathrm{~ms}$.

The spectra shown in Figure 4 and 5 prove that all modes are damped.

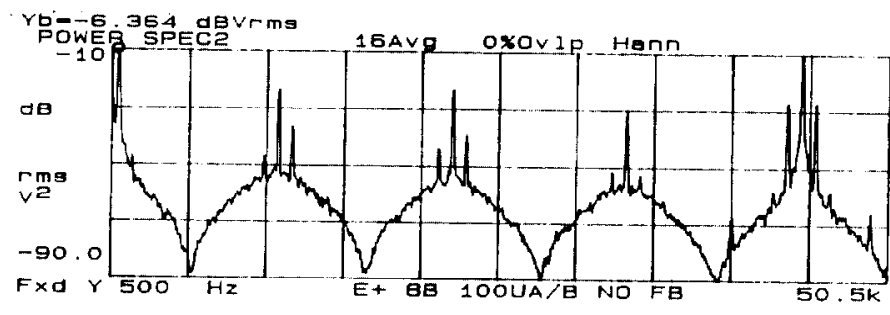

Figure 4. Spectrum of the dipole oscillations for an eight bunch positron beam of $100 \mu \mathrm{A}$ per bunch. The synchrotron frequency is $875 \mathrm{~Hz}$ and all eight modes are seen as sidebands to the revolution frequency of $11.25 \mathrm{kHz}$ and its harmonics. The largest amplitude is measured on the mode 0 at $875 \mathrm{~Hz}$, followed by mode 4 seen at 44.1 and $45.86 \mathrm{kHz}$.

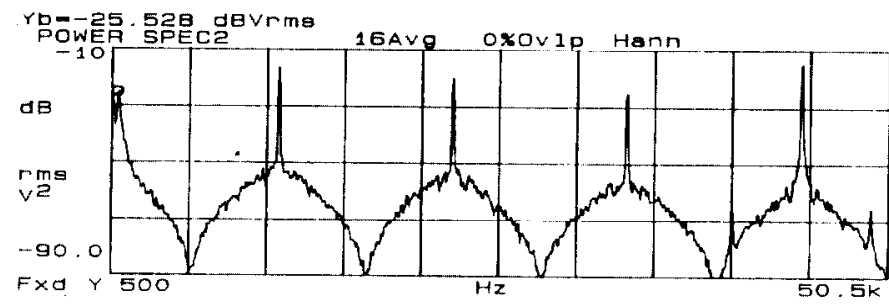

Figure 5. The spectrum seen in Figure 4 measured with longitudinal fccdback and a voltage of $1.2 \mathrm{MV}$. All eight modes are damped. The remaining spectral lines are the revolution frequency and its first three harmonics.

The system is now used routinely during accumulation at $20 \mathrm{GeV}$ with a feedback voltage of $1.2 \mathrm{MV}$ which requires a klystron output power of $90 \mathrm{~kW}$. After acceleration to the collision energy of about $45.6 \mathrm{GeV}$ where longitudinal feedback is not required the system is switched off.

\section{ACKNOWLEDGEMENTS}

The rapid installation and commissioning of the longitudinal feedback system for LEP was due in large part to the use of cavities, waveguide pieces and klystron from the Deutsches Elektronen-Synchrotron (DESY) laboratory. The authors would like to thank the DESY laboratory for providing this equipment and also to thank the members of the DESY MHF group for their collaboration and support.

\section{REFERENCES}

[1] J. C. Juillard and E. Peschardt, "Damping of Longitudinal Coupled Bunch Oscillations in LEP", Proc. 2nd EPAC, Nice, June 1990, pp. 1554-56.

[2] R. Bailey, J. M. Jowett, W. Kalbreier and D. Wang, "Commissioning and operation of the LEP Pretzel scheme", this conference, paper Pb5.

[3] S. Myers, "A First Look at Longitudinal Feedback for LEP", LEP note 366.

[4] P. Brown, E. Ciapala, S. Hansen, E. Peschardt and J. Sladen, "The LEP radio-frequency low power system", IEEE Trans. Nucl. Sci., NS-23, October 1985, pp. 2032-34. 